\title{
Multi-Objective Synthesis of Filtering Dipole Array Based on Tuning-Space Mapping
}

\author{
Petr VSETULA, Zbynek RAIDA, Jaroslav LACIK \\ Dept. of Radio Electronics, Brno University of Technology, Technicka 12, 61600 Brno, Czech Republic \\ raida@feec.vutbr.cz
}

\begin{abstract}
In the paper, we apply tuning-space mapping to multi-objective synthesis of a filtering antenna. The antenna is going to be implemented as a planar dipole array with serial feeding. Thanks to the multi-objective approach, we can deal with conflicting requirements on gain, impedance matching, side-lobe level, and main-lobe direction. MOSOMA algorithm is applied to compute Pareto front of optimal solutions by changing lengths of dipoles and parameters of transmission lines connecting them into a serial array. Exploitation of tuning space mapping significantly reduces CPU-time demands of the multi-objective synthesis: a coarse optimization evaluates objectives using a wire model of the filtering array (4NEC2, method of moments), and a fine optimization exploits a realistic planar model of the array (CST Microwave Studio, finite integration technique). The synthesized filtering antenna was manufactured, and its parameters were measured to be compared with objectives. The number of dipoles in the array is shown to influence the match of measured parameters and objectives.
\end{abstract}

\section{Keywords}

Dipole antenna array, filtering antenna, filtenna, multi-objective optimization, tuning-space mapping

\section{Introduction}

In communication engineering, a transmit antenna can be understood as a two-port network which transforms a guided wave on an input port to a radiated wave on an output port. Such a two-port network can be described by scattering parameters [1], [2]:

- Reflection coefficient at the input $S_{11}$ is related to the power reflected back to the source by impedance mismatch of the input port (under the condition that the output port is matched).

In antenna engineering, frequency response of reflection coefficient can define impedance bandwidth of the antenna $\Delta f_{i m}$

$$
\left|S_{11}(f)\right|<-10 \mathrm{~dB}, \quad f \in \Delta f_{\text {im }} .
$$

- Transmission coefficient $S_{21}$ is related to the power transmitted from an input port to an output port of the network (under the condition that the output port is matched).

In filter engineering, frequency response of transmission coefficient can define selectivity of the filter [2]

with

$$
S=\frac{\Delta f_{-3 \mathrm{~dB}}}{\Delta f_{-60 \mathrm{~dB}}}
$$

$$
\begin{aligned}
& \left|S_{21}(f)\right|>-3 \mathrm{~dB}, \quad f \in \Delta f_{-3 \mathrm{~dB}}, \\
& \left|S_{21}(f)\right|>-60 \mathrm{~dB}, \quad f \in \Delta f_{-60 \mathrm{~dB}} .
\end{aligned}
$$

In antenna engineering, transmission coefficient can be related to the gain of an antenna in a main-lobe direction (a transmission from the input port to the main-lobe direction is considered). Therefore, frequency response of gain can define selectivity of an antenna playing the secondary role of a frequency filter.

An ideal band-pass filter exhibits a constant transmission coefficient in the pass-band, and a high selectivity. If an antenna behaves similarly (a constant gain in the pass-band and a high selectivity are achieved), we eliminate the need of using the band-pass filter at the output of the receive antenna because the corresponding filtering is performed by the antenna itself.

Antennas performing radiation and frequency filtering simultaneously are called filtering antennas (or filtennas) [3], [4]. Existing approaches to the synthesis of filtennas are based on following approaches:

- An antenna element is represented by an equivalent circuit, and the circuit is optimized to obtain desired filtering properties. In [5] and [6], specific planar radiators are synthesized to obtain a prescribed frequency response of reflection coefficient at the input of the antenna. Radiation properties are not included in the synthesis.

- A planar filter is designed in the first step. Then, the output port is removed, and the filter structure is modified to support the radiation [7]. Hence, the filter design methodology is used to develop an antenna, 
but radiation parameters are not considered during the design again.

- A feeding network of an antenna array is designed such a way so that the main-lobe direction stays constant in the pass-band. The design of antenna elements follows an equivalent-circuit approach to obtain requested filtering properties [8]. Nevertheless, radiation and filtering objectives are met separately during the design.

In our approach, we understand an array of dipoles with serial feeding being a frequency filter:

- An input port of the filter corresponds to the input port of the antenna. An output port of the filter corresponds to the radiation of the antenna in main lobe direction.

- Dipoles in the antenna array correspond to shunt resonators in the structure of the filter.

- In the pass-band, the antenna array is in resonance, and maximum of the excitation power is radiated to the main-lobe direction. In the stop-band, the excitation power is reflected by the input port of the detuned antenna back to the source.

In the role of frequency filter, the antenna array is described by frequency response of reflection coefficient as the first objective and frequency response of gain in the main-lobe direction (an equivalent of the transmission coefficient) as the second objective.

Nevertheless, an antenna array behaves as a spatial filter at the same time. Spatial properties of the antenna can be described by frequency response of sidelobe level as the third objective and deflection of the main-lobe direction as the fourth objective.

Synthesis of filtennas can be therefore conceived as a multi-objective optimization problem. According to our knowledge, such an approach to the synthesis of filtennas has not been published in open literature yet. The design process presented in this paper meets two radiation objectives (maximum sidelobe level, minimum main-lobe deflection) and two filtering objectives (maximum main-lobe gain, minimum reflection coefficient) together.

In order to reduce fabrication costs and ensure repeatability of production, the dipole filtenna is expected to be manufactured by a planar technology. Numerical analysis of a realistic planar antenna is relatively time-consuming since the complicated antenna structure has to be modeled by differential techniques. In order to reduce CPU-time costs, tuning-space mapping is applied [9].

The mapping consists of two stages:

- Coarse optimization. Partial objectives are evaluated using a numerical model of an array of wire dipoles connected by serial feeding lines. Such a model of an antenna can be easily implemented in 4NEC2 (the method of moments).
- Fine optimization. Results of the coarse optimization are tuned using a realistic model of a planar dipole array implemented in CST Microwave Studio (the finite integration technique).

That way, CPU-time demands of the multi-objective synthesis of a planar dipole filtenna can be minimized. Most of iterations are performed using a CPU-moderate 4NEC model, and a low number of iterations are done using a CPU-expensive CST model. The synthesis process is described in Sec. 2 of the paper in detail.

In Sec. 3, parameters of the synthesized array are verified experimentally. Attention is dominantly turned to frequency responses of reflection coefficient, gain, side lobe level, and main lobe direction which were used to formulate objectives. Nevertheless, the synthesized filtering array is an antenna, and therefore directivity patterns of co-polar components and cross-polar ones are important as well. We therefore present results of measurements of those patterns as well.

Section 4 concludes the paper.

\section{Synthesis of Dipole Array with Serial Feeding}

The synthesized antenna array consists of parallel dipoles placed above the ground plane which limits the radiation to a half-space and increases the gain of the array. The array can contain 8,16 or 32 dipoles.

In [10], we compared various configurations of arrays of wire dipoles to be synthesized. From the fabrication viewpoint, dipoles connected by serial transmission lines were identified as a suitable structure.

The synthesized filtenna should behave like a frequency filter with the pass-band from $2.9 \mathrm{GHz}$ to 3.1 GHz:

- In the pass-band, we require gain in main lobe direction $G_{\text {pass }}>13 \mathrm{dBi}$, reflection coefficient $S 11_{\text {pass }}<-12 \mathrm{~dB}$, and sidelobe level $S L L_{\text {pass }}>6 \mathrm{~dB}$. Finally, we accept the main lobe deflection $\theta= \pm 5^{\circ}$.

- In the stop-band, we require gain in the main lobe direction $G_{\text {stop }}<5 \mathrm{dBi}$, reflection coefficient $S 11_{\text {stop }}>-5 \mathrm{~dB}$, and sidelobe level $S L L_{\text {stop }}<2 \mathrm{~dB}$.

In the synthesis of a planar dipole array with prescribed filtering capabilities, we use two different numerical models of the filtenna:

- Surrogate model of the array of wire dipoles with serial feeding and a ground plane (4NEC2, moment method).

- Realistic model of the array of planar dipoles with serial feeding, a balun and a ground plane (CST Microwave Studio, finite integration techniques). The realistic model of the filtenna is depicted in Fig. 1. 


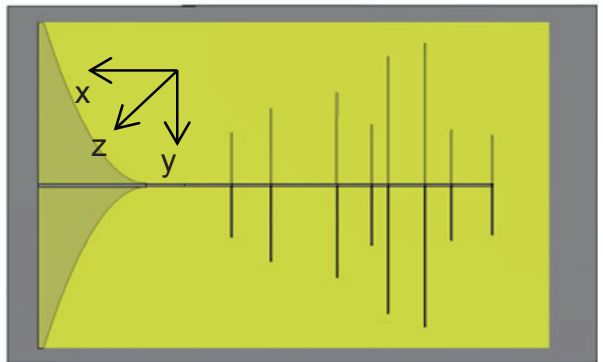

Fig. 1. Top view of the planar array of 8 dipoles with serial feeding. Dark gray: top layer (the left arm of dipoles and serial feeding). Light gray: bottom layer (the right arm of dipoles, serial feeding and symmetrization). Ground plane (grey) is placed below the substrate (yellow).

The optimizer changes amplitudes and phases of excitation currents, distances between dipoles and lengths of dipoles.

Amplitudes and phases of excitation currents are not expressed in the optimization formulation explicitly. Amplitudes and phases of currents are formed by serial feeders and are influenced by input impedances of dipoles in the array.

State variables (distances between dipoles, lengths of dipoles, and parameters of serial feeders) are iteratively computed by the multi-objective self-organizing migration algorithm (MOSOMA) [11]. For conflicting objectives, MOSOMA is able to determine the Pareto front of optimal solutions.

MOSOMA is aimed to meet four objectives: (1) maximum gain in the main lobe direction, (2) maximum sidelobe level, (3) minimum reflection coefficient at the input, and (4) minimum main lobe deflection.

In Fig. 2, two-dimensional cut of the four-dimensional objective space is depicted. The horizontal axis $f_{2}$ is related to the functional value of the sidelobe-level objective, and the vertical axis $f_{1}$ corresponds to the functional value of the gain objective. The set of evaluated non-optimal solutions is represented by blue points, and the optimal solutions forming the Pareto front are indicated by red points.

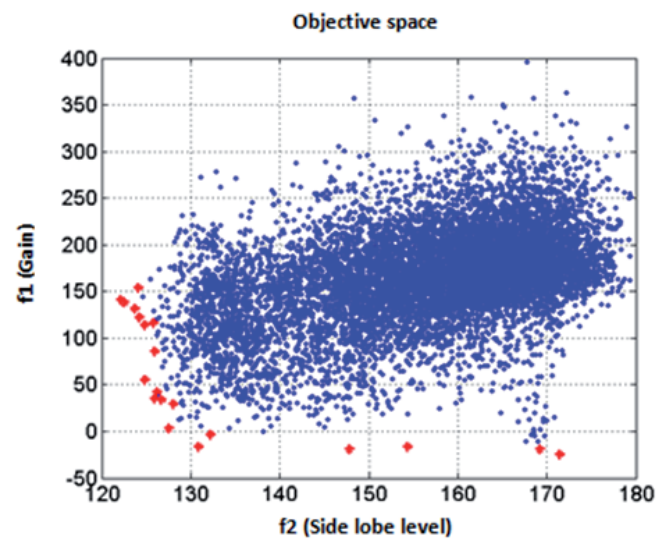

Fig. 2. Two-dimensional cut of the four-dimensional objective space the filtering antenna is optimized in. Horizontal: functional value of the sidelobe-level objective. Vertical: functional value of the gain objective.
A high number of evaluated solutions show that the exploitation of the CPU-effective 4NEC surrogate model of the filtenna is reasonable. The 4NEC model is used to reveal dominating solutions creating the Pareto front (red points). Consequently, the dominating solutions are refined using the realistic CST model of the filtenna (Fig. 1).

Figure 3 shows results of the coarse optimization of the surrogate model of the filtenna (wire dipoles in 4NEC):

- Impedance matching achieves good values around the resonant frequency (Fig. 3a).

- Frequency responses of gain show that selectivity of the filtenna (the slope of gain) depends on the number of dipoles (Fig. 3b).

- Requested main lobe deflection is met by 8 and 32 dipoles only (Fig. 3c).

- Requested sidelobe level is not reached.

The coarse optimization produces the dominating solutions in the form of state vectors. Distances between wire dipoles, and lengths of wire dipoles are elements of these state vectors.

When converting the wire filtenna into a planar one:

- Distances between planar dipoles were set to be identical with the distances between wire dipoles.

- Lengths of planar dipoles were set to be identical with the lengths of wire dipoles.

- Widths of planar dipoles were set to be identical with diameters of wire dipoles.

- The serial feeder connecting dipoles was designed with the characteristic impedance $Z_{0}=73 \Omega$ corresponding to input resistance of a dipole in resonance.

For the implementation of the converted planar layout, we used a thin substrate ARLON CuClad 217 with a low dielectric constant $\varepsilon_{r}=2.17$ and thickness $t=0.508 \mathrm{~mm}$.

That way, an input of the fine optimization (planar dipoles in CST Microwave Studio) was created.

In the first step, we design a transformer [microstrip transmission line $\rightarrow$ [balanced stripline] (balun). We have adopted a compact balun published in [12].

In order to implement the impedance matching, we insert an impedance transformer between the balun and the array. In our case, a $\lambda / 4$ impedance transformer is used.

For simplicity, let us concentrate on an array consisting of 8 dipoles. In Fig. 4:

- Results of the coarse optimization are represented by red line and depicted as 4NEC (identical with blue dependencies in Fig. 3).

- Results of the initial CST model created by converting the 4NEC wire model are represented by blue line and depicted as CST.

- Results of the fine optimization are represented by green line and depicted as CST opt. 


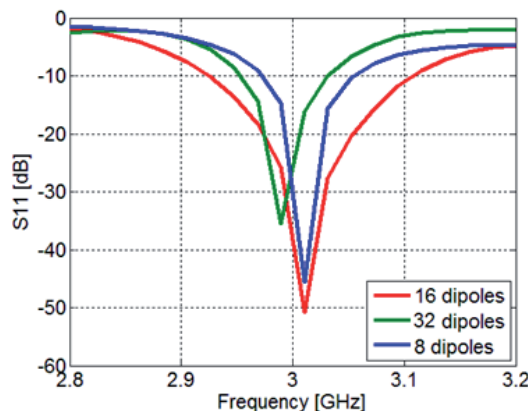

(a)

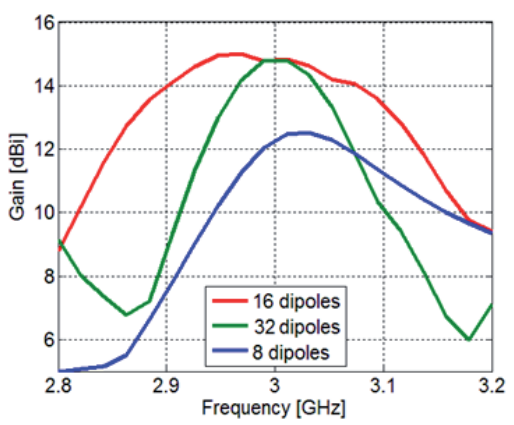

(b)

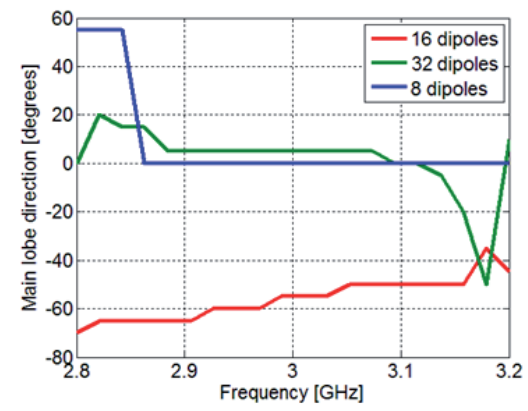

(c)

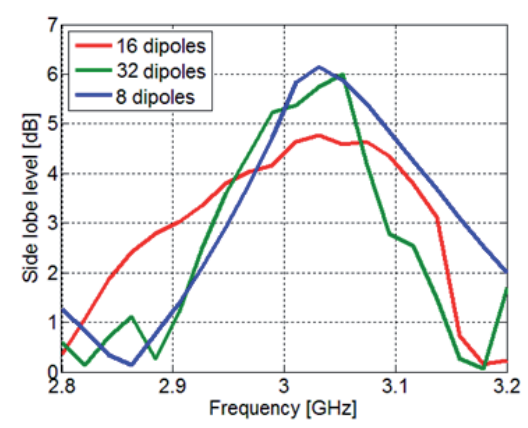

(d)

Fig. 3. Outputs of 4 NEC simulations of wire array with seria feeding consisting of 8 dipoles (blue), 16 dipoles (red) and 32 dipoles (blue). Frequency response of return loss (a), gain (b), main lobe direction (c), side lobe level (d).

The conversion of the wire filtenna to the planar one degrades parameters of the filtering antenna (red lines versus blue ones in Fig. 4). Nevertheless, the degradation can be efficiently eliminated by a local tuning of the realistic CST model. Parameters of the fine planar model after

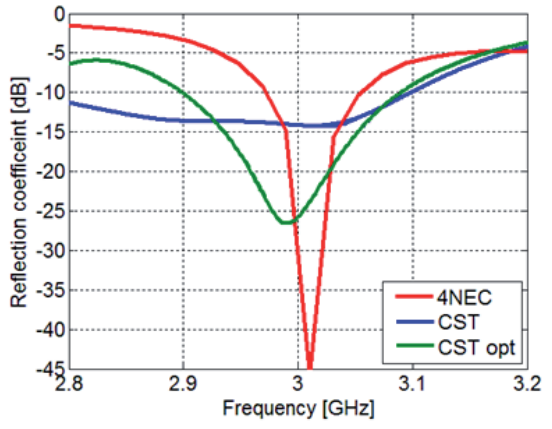

(a)

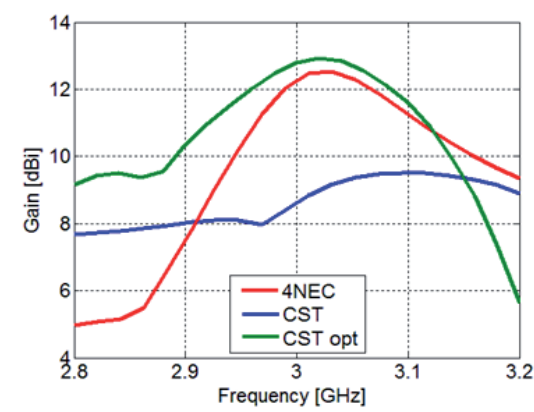

(b)

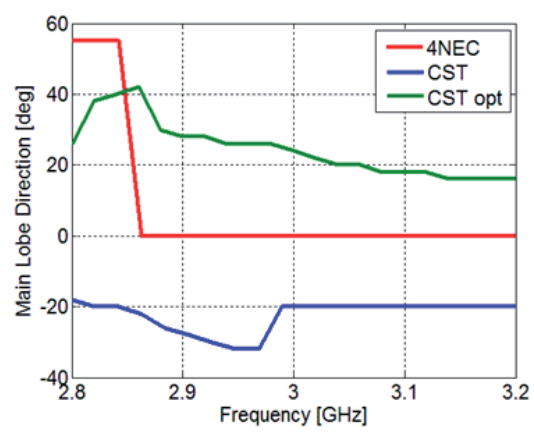

(c)

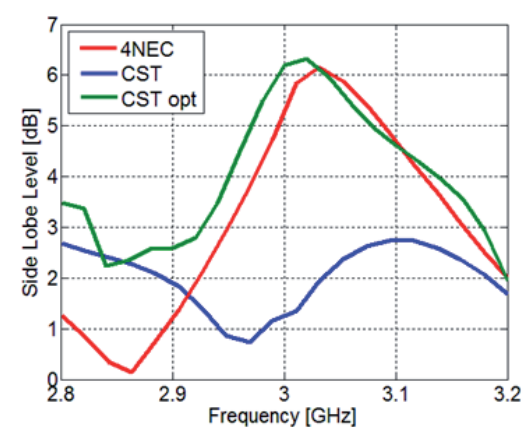

(d)

Fig. 4. Antenna array with serial feeding consisting 8 dipoles. The 4NEC simulation of wire array (4NEC, red). Initial CST simulation of planar array (CST, blue). Final CST simulation of optimized planar array (CST opt., green). Frequency response of reflection coefficient (a), gain (b), main lobe direction (c), sidelobe level (d).

tuning (green lines in Fig. 4) approach parameters of the coarse wire surrogate model (red lines in Fig. 4).

The planar model exhibits: 
- Better impedance bandwidth $\mathrm{S}_{11}<-10 \mathrm{~dB}$ (Fig. 4a). Whereas the wire antenna is matched from $2.97 \mathrm{GHz}$ to $3.06 \mathrm{GHz}$, the planar filtenna meets the required band from $2.90 \mathrm{GHz}$ to $3.10 \mathrm{GHz}$.

- Higher gain (Fig. 4b). The gain approaches the required value $G_{\text {pass }}=13 \mathrm{dBi}$ in the maximum. In the stop-band, gain approaches $G_{\text {stop }}=5 \mathrm{dBi}$.

- The main lobe deflection exceeds the requested value $\theta= \pm 5^{\circ}$ (Fig. 4c). Here, further effort is needed.

- Higher sidelobe level (Fig. 4d). The sidelobe level corresponds to the required value $S L L_{\text {pass }}=6 \mathrm{dBi}$ in the maximum. In the stop-band, sidelobe level approaches $S L L_{\text {stop }}=2 \mathrm{dBi}$.

We can therefore conclude that the optimization of the surrogate model helps to reveal optima of the realistic one.

Since the described synthesis process of filtering antennas is not able to influence the effect of losses, we have neglected all the losses both in the CPU-effective 4NEC model and the realistic CST model.

Moreover, losses are very small in both the cases:

- The wire antenna can be constructed from metallic conductors in air. Therefore, losses in metal can influence parameters of the antenna only.

- The planar antenna consists of metallic strips on both sides of a very thin dielectric substrate. Since a negligible portion of electromagnetic field propagates through the substrate only, the planar structure exhibits similar losses like the wire one.

The effect of losses can be described by the efficiency of the antenna or the realized gain. All the gains given in the paper are realized ones.

\section{Experimental Verification}

We fabricated the dipole array of 8 dipoles with the serial feeding from the substrate ARLON CuClad 217 (Fig. 5). The planar antenna was equipped with an SMA connector. We completed the dipole array by the planar reflector of finite dimensions $160 \mathrm{~mm} \times 260 \mathrm{~mm}$ from the tinplate. The distance between the planar reflector and the dipole array was $\lambda / 4$. As standoffs, we used polystyrene foam bricks.

The planar reflector limits the radiation to the halfspace above the antenna which positively influences the gain of the antenna (see Fig. 6). At the central frequency $3 \mathrm{GHz}$, the antenna exhibited the realized gain $8 \mathrm{~dB}$ without the reflector (red line) and $13 \mathrm{~dB}$ with an infinite reflector (blue line).

In numerical simulations, we have also verified the effect of finite dimensions of the reflector (green line in Fig. 6). Obviously, the dimensions $160 \mathrm{~mm} \times 260 \mathrm{~mm}$ of the finite reflector are sufficient because frequency response of both the gains are close each other.

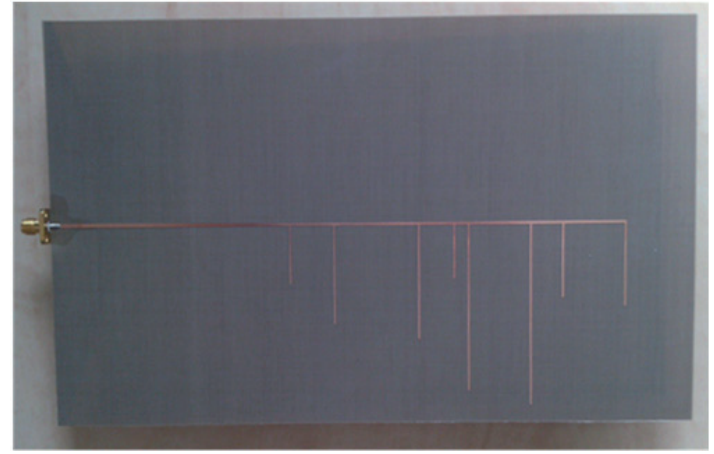

(a)

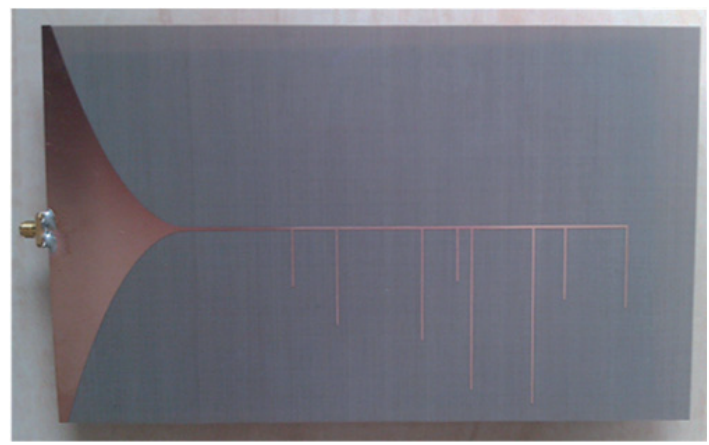

(b)

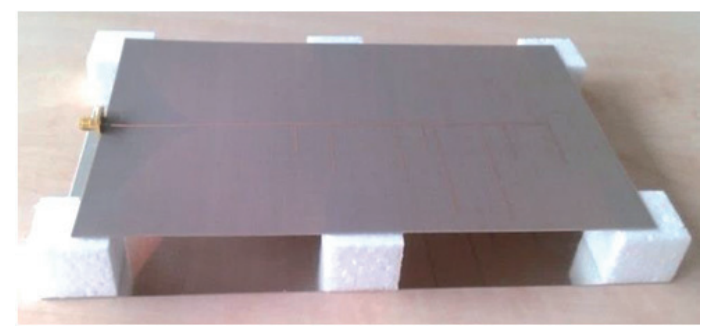

(c)

Fig. 5. Prototype of the manufactured planar array of 8 dipoles with serial feeding and the ground plane. Top view (a), bottom view (b), side view (c).

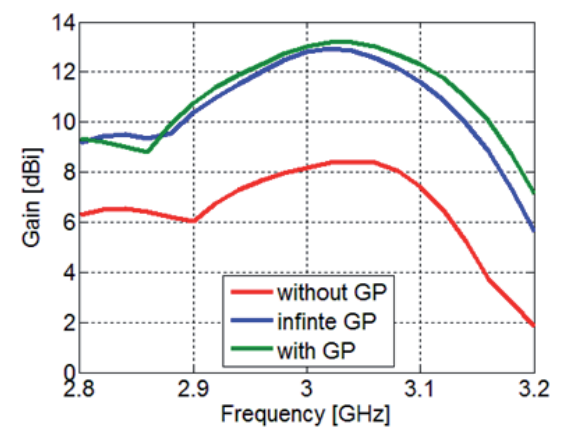

Fig. 6. Frequency response of realized gain of 8 dipoles with serial feeding without reflector (red), with infinite reflector (blue) and with finite reflector (green).

In Fig. 7a, we compare the simulated reflection coefficient and the measured one at the input of the filtenna. The resonant frequency of the measured antenna is slightly lower compared to the simulated one. Correspondingly, the maximum value of the side lobe level and the main lobe direction are also shifted to lower frequencies. 


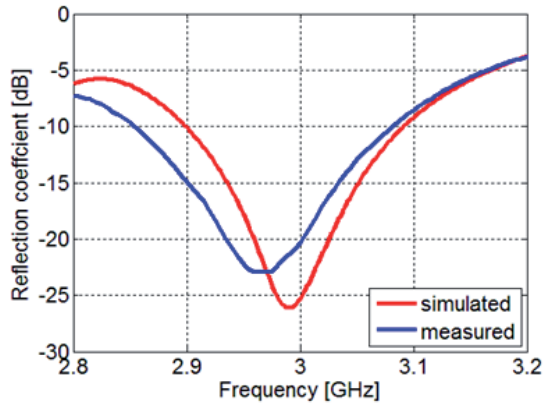

(a)

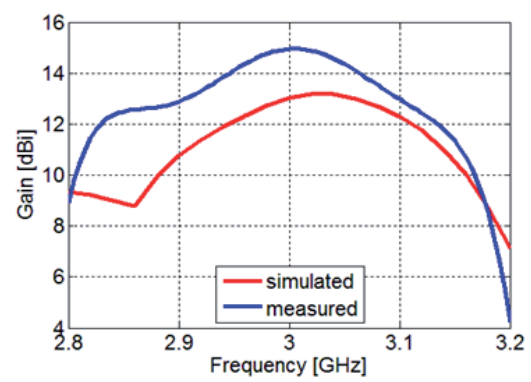

(b)

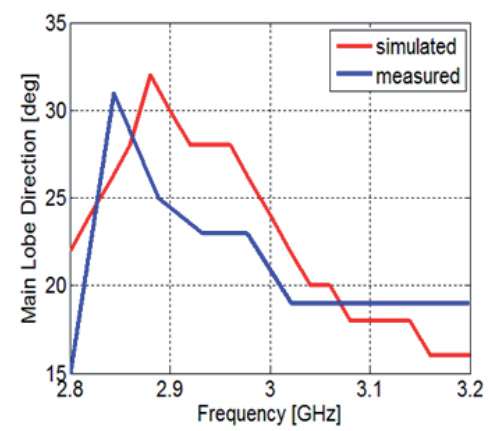

(c)

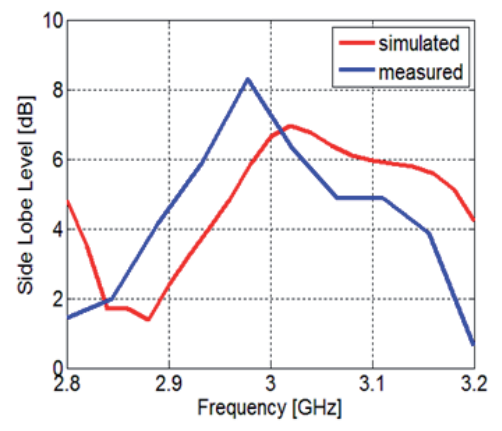

(d)

Fig. 7. Planar array of 8 dipoles with serial feeding: simulated parameters (red) versus measured ones (blue). Frequency response of reflection coefficient (a), gain (b), main lobe direction (c) and side lobe level (d).

The frequency shift is probably caused by simplifications in the numerical model of the planar array (perfect conductivity, neglected polystyrene bricks, a simplified model of the coaxial connector, etc.).

The fabricated dipole array achieved a worse value of the reflection coefficient in minimum. On the other hand, the bandwidth of the array was slightly increased.

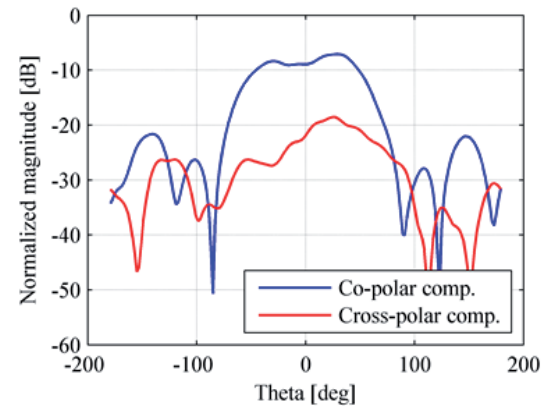

(a)

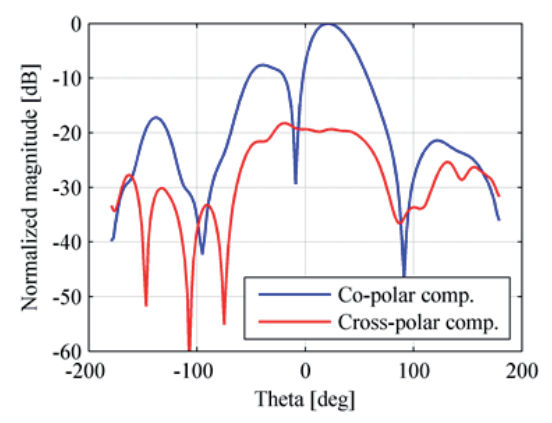

(b)

Fig. 8. Normalized directivity patterns of the filtering array measured at the central frequency $f=3 \mathrm{GHz}$. Co-polar components (blue), cross-polar components (red). E-plane (a), H-plane (b).

The comparison of the simulated and measured gain is depicted in Fig. 7b. Clearly, the measured gain is higher than the simulated one. When depicting Fig. 7b, we measured the gain at ten frequencies in the range from $2.8 \mathrm{GHz}$ to $3.2 \mathrm{GHz}$. Then, we interpolated values of the measured gain by a polynomial (the blue one). The measured dipole array exhibited a better selectivity, but in a wider range of frequencies.

Except of slight frequency shifts, frequency responses of the main lobe direction and side lobe level are very similar (see Fig. 7c and 7d).

In order to characterize directive properties of the synthesized filtering array, normalized directivity patterns were measured at the central frequency $f=3 \mathrm{GHz}$. In Fig. 8a, co-polar and cross-polar components in E-plane are shown. In Fig. 8b, both the components in H-plane are given.

In the so-far characterization, the realized gain in the main lobe direction was considered as the dominant objective of the synthesis. The prescribed side lobe level and the prescribed main lobe direction played the role of constraints for the synthesis in a fact.

\section{Summary}

In the paper, we described a multi-objective synthesis of a filtering antenna consisting of planar dipoles. Frequency responses of gain, reflection coefficient, sidelobe level and main-lobe deflection were objectives of the optimization. 
Pareto front of optimal solutions was computed by the multi-objective self-organizing migration algorithm (MOSOMA). Since the computation of the Pareto front is CPUtime expensive, the accurate numerical model of a planar filtenna was replaced by a surrogate model of a wire antenna for coarse optimization. Results approve functionality of this approach which is an implementation of a tuning-space mapping in a fact.

The synthesized filtering antenna met requirements on the frequency response of gain, impedance matching and sidelobe level. Main-lobe deflection was higher than the prescribed one.

The designed antenna was fabricated and measured. Results of measurements confirmed validity of simulations.

\section{Acknowledgments}

Research described in this paper was financed by the Czech Ministry of Education under grant LD12012. Research is a part of the COST Action IC 1102 VISTA. For simulations and measurements, infrastructure of the SIX Center was used.

\section{References}

[1] HONG, J. S., LANCASTER, M. J. Microstrip Filters for RF/Microwave Applications. New York: John Wiley \& Sons, 2001.

[2] DIMOPOUlos, H. G. Analog Electronic Filters: Theory, Design and Synthesis (Analog Circuits and Signal Processing). Dordrecht: Springer, 2012.

[3] BAILEY, M. C. A stacked patch antenna design with strict band pass filter characteristics. In Proceedings of the APS International Symposium. Monterey (USA), 2004, p. 1599-1602. DOI: 10.1109/APS.2004.1330498

[4] WANG, Y. P., HALl, P. S., GARDNER, P., WU, J. H. Yagi antenna with improved out-of-band gain suppression. Electronics Letters, 2012, vol. 48, no. 10, p. 564-548. DOI: $10.1049 / \mathrm{el} .2012 .0351$

[5] ABUNJAIleH, A. I., HUNTER, I. C., KEMP, A. H. A circuittheoretic approach to the design of quadruple-mode broadband microstrip antennas. IEEE Transactions on Microwave Theory and Techniques, 2008, vol. 56, no. 4, p. 896-900. DOI: 10.1109/TMTT.2008.918137

[6] LIN, C. K., CHUNG, S. J. A compact filtering microstrip antenna with quasi-elliptic broadside antenna gain response. IEEE Antennas and Wireless Propagation Letters, 2011, vol. 10, p. 381-384. DOI: 10.1109/LAWP.2011.2147750

[7] WU, W. J., YIN, Y. Z., ZUO, S. L., ZHANG, Z. Y., XIE, J. J. A new compact filter-antenna for modern wireless communication systems. IEEE Antennas and Wireless Propagation Letters, 2011, vol. 10, p. 1131-1134. DOI: 10.1109/LAWP.2011.2171469

[8] LIN, C. K., CHUNG, S. J. A filtering microstrip antenna array. IEEE Transactions on Microwave Theory and Techniques, 2011, vol. 59, no. 11, p. 2856-2863. DOI: 10.1109/TMTT.2011.2160986
[9] KOZIEL, S., MENG, J., BANDLER, J. W., BAKR, M. H., CHENG, Q. S. Accelerated microwave design with tuning space mapping. IEEE Transactions on Microwave Theory and Techniques, 2009, vol. 57, no. 2, p. 383-394. DOI: 10.1109/TMTT.2008.2011313

[10] VSETULA, P., RAIDA, Z. Dipole antenna array with synthesized frequency dependency of gain and reflection coefficient. In Proceedings of the ICEAA 2013 Conference. Torino (Italy), 2013, p. 1087-1090. DOI: 10.1109/ICEAA.2013.6632410

[11] KADLEC, P., RAIDA, Z. A novel multi-objective self-organizing migrating algorithm. Radioengineering, 2011, vol. 20, no. 4, p. 804-815.

[12] ABBosh, A. M., KAN, H. K., BIALKOWSKI, M. E. Compact ultra-wideband planar tapered slot antenna for use in a microwave imaging system. Microwave and Optical Technology Letters, 2006, vol. 48, no. 11, p. 2212-2216. DOI: 10.1002/mop.21906

[13] KOZIEL, S., CHENG, Q. S., BANDLER, J. W. Space mapping. IEEE Microwave Magazine, 2008, vol. 9, no. 6, p. 105-122. DOI: 10.1109/MMM.2008.929554

\section{About the Authors...}

Petr VŠETULA was born in Kyjov, Czech Republic, in April 1985. He graduated from the Faculty of Electrical Engineering and Communication (FEEC), Brno University of Technology (BUT), in 2014 when he received the Ph.D. degree. $\mathrm{He}$ is interested in small antennas and arrays design.

Zbyněk RAIDA has graduated at Brno University of Technology (BUT), Faculty of Electrical Engineering and Communication (FEEC). Since 1993, he has been with the Dept. of Radio Electronics FEEC BUT, now as a Professor. In 1996 and 1997, he was with the Laboratoire de Hyperfrequences, Universite Catholique de Louvain, Belgium, working on variational methods of numerical analysis of electromagnetic structures. In 2006-2013, he was the head of the Dept. of Radio Electronics, FEEC BUT, and since 2010 he has been the head of the SIX Center. Together with his students and colleagues, Zbyněk Raida has been working on numerical modeling and optimization of electromagnetic structures, exploitation of artificial neural networks for solving electromagnetic compatibility issues, and the design of special antennas. Zbyněk Raida is a member of IEEE Microwave Theory and Techniques Society.

Jaroslav LÁČ́ÍK received the M.Sc. and Ph.D. degrees from Brno University of Technology, Brno, Czech Republic, in 2002 and 2007, respectively. He is currently an Associate Professor at the Dept. of Radio Electronics, Faculty of Electrical Engineering and Communication, Brno University of Technology. His research interests are antennas, body-centric wireless communication, and computational electromagnetics. He is a member of IEEE. 